\title{
DAMAGE ESTIMATION OF EXPLOSIONS IN URBAN ENVIRONMENTS BY SIMULATION
}

\author{
Ludwig Mohr ${ }^{1}$, Richard Benauer ${ }^{2}$, Peter Leit ${ }^{2}$, Friedrich Fraundorfer $^{1}$ \\ ${ }^{1}$ Institute of Computer Graphics and Vision, Graz University of Technology, 8010 Graz, Austria \\ ludwig.mohr@icg.tugraz.at, fraundorfer@icg.tugraz.at \\ ${ }^{2}$ Bionic Surface Technologies GmbH, 8010 Graz, Austria benauer@bionicsurface.com, leitl@bionicsurface.com
}

KEY WORDS: Blast Wave, Semantic 3D Modeling, Computational Fluid Dynamics, Damage Estimation

\begin{abstract}
:
Precise models of the impact of explosions in urban environments provide novel and valuable information in disaster management for developing precautionary, preventive and mitigating measures. Yet to date, no methods enabling accurate predictions of the process and effect of detonations at particular locations exist. We propose a novel approach mitigating this gap by combining state-of-the-art methods from photogrammetric 3D reconstruction, semantic segmentation and computational based numerical simulations. In a first step, we create an accurate urban 3D reconstruction from georeferenced aerial images. The resulting city model is then enriched with semantic information obtained from the original source images as well as from registered terrestrial images using deep neural networks. This allows for an efficient automatic preparation of a 3D model suitable for the use as a geometry for the numerical investigations. Using this approach, we are able to provide recent and precise models of an area of interest in an automated fashion. Within the model, we are now able to define the explosive charge size and location and simulate the resulting blast wave propagation using CFD simulation. This provides a full estimation for the expected pressure propagation of a defined charge size. From these results, arising damages and their extent, as well as possible access routes or countermeasures, can be estimated. Using georeferenced sources allows for the integration and utilization of simulation results into existing geoinformation systems of disaster management units, providing novel inputs for training, preparation and prevention. We demonstrate our proposed approach by evaluating expected glass breakage and expected damages impairing the structural integrity of buildings depending on the charge size using a 3D reconstruction from aerial images of an area in the inner city of Graz, Austria.
\end{abstract}

\section{NOMENCLATURE}

\section{Abbreviations}

SfM Structure from Motion

CNN Convolutional Neural Network

CFD Computational Fluid Dynamics

FEM Finite Element Method

RANS Reynolds-Averaged Navier-Stokes

TNT Trinitrotoluene explosive

\section{Symbols}

$E_{T N T}$ Energy of an explosive charge

$M_{T N T}$ TNT mass equivalent of an explosive charge

$\gamma$ Adiabatic index of air

$V_{b a l}$ Volume of a compressed gas filled sphere

$p_{0}$ Initial ambient total pressure

Ma Mach speed

\section{INTRODUCTION}

Accurate estimation of the effect of an explosion is an essential prerequisite for effective and meaningful preparation and training for an emergency. Yet, especially in urban environments, this necessary data is hard to come by, since field tests are infeasible, empirical methods for estimating the expected effect and damage radius of an explosion on the other hand only produce inaccurate results. One of the reasons for this

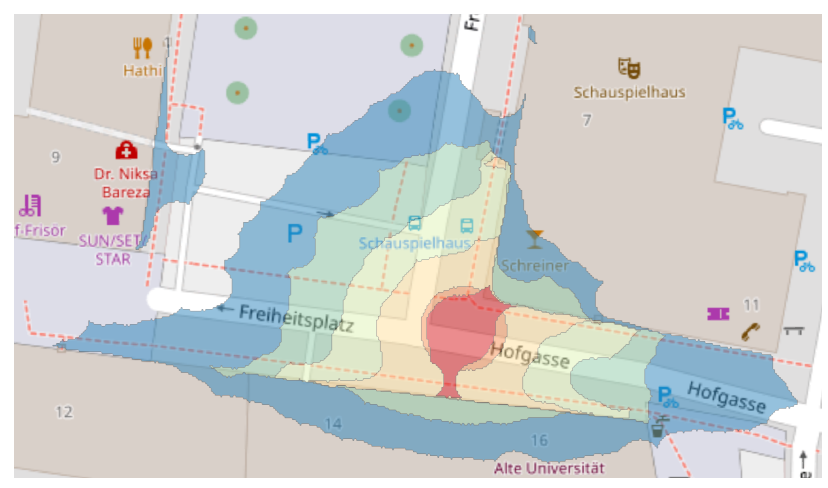

Figure 1. Example of the result of a simulation of an explosive charge with $10 \mathrm{~kg}$ of equivalent mass prepared for direct integration into a GIS solution.

is their reliance on a free field assumption for the propagation of the shock wave, thereby ignoring effects in reflections at complex geometry present in urban environments. Research on methods for more precise numerical estimation of shock wave propagation able to consider geometric constraints exist at least 
since the 1950ies (Brode, 1955). Today, with the availability of large scale numerical simulation in combination with potent numerical solvers, at least in theory, nothing stands in the path of performing accurate analysis of shock wave propagations of explosive expansions in complex geometries. Yet, it still remains an open question how to acquire intermediate-scale or large-scale scene geometries suitable for numerical evaluation without having to perform time consuming manual editing. On the other hand, reconstruction of the underlying 3D scene geometry from both aerial and terrestrial image data is an active area of research in the photogrammetry community, where a large body of methods tackling this task exists. Balancing the demands for highly detailed and complete reconstructions which capture the necessary scene complexity with the needless increase in computational complexity of the simulation by introduction of scene details which are irrelevant or insignificant to an explosive expansion is still an open question. Furthermore, the question of how to present the results of such a simulation in a relevant and usable way to disaster management forces for preparation and training is yet unsolved.

In this work, we propose an approach uniting the fields of photogrammetric 3D reconstruction and numerical simulation of explosive expansions and demonstrate ways to make results of such simulations directly available to disaster management by integrating them in existing GIS solutions (cf. Figure 1). We start by giving a short overview over previous works done in the fields of photogrammetric 3D reconstruction and semantic segmentation as well as simulation and damage categorization of explosions in section 2, and continue by presenting our proposed approach in sections 3 and 4 . Possibilities to make the results of the simulation usable for disaster management units and emergency services by integrating them seamlessly in existing GIS solutions is detailed in section 5. We finally present the results of a case study performed in the city of Graz, Austria, in section 6 . The paper concludes in section 7 with a summary and an outlook.

All figures are best viewed at high resolution in the digital version of this paper on the computer.

\section{RELATED WORK}

Simulating explosions by modelling the explosive charge as an initial volume filled with compressed gas is well-known and has been employed in many projects (Brode, 1955, Larcher, Casadei, 2010). Other work has already successfully applied this approach for blast wave calculations with geometries (Sohaimi et al., 2016). In contrast to their work, which only uses small geometric structures or free fields, we demonstrate its use in a large scale reconstruction of an urban environment. In contrast to FEM modeling (Fairlie, 1998), this model provides enough precision for medium and long distances without a need for increasing computational costs.

Structure from Motion (SfM), or the joint 3D reconstruction of geometric structures and estimation of camera poses from a set of images, is a well researched topic in the computer vision community (Hartley, Zisserman, 2000) with an ever increasing number of algorithms and improvement to overall robustness (Özyeşil et al., 2017) which has lead to several readily available software solutions for 3D reconstruction, both free and commercial (Schönberger, Frahm, 2016, Agisoft, 2019, Mapillary, 2017). If the aim is automated editing of a reconstructed scene, it is essential to know which parts of the scene reconstruct which class of objects. In Computer Vision, solving this task automatically for each data point is known as semantic segmentation. In recent years, the advent of deep-learning based methods utilizing CNNs has revolutionized this field, virtually all conventional approaches to semantic segmentation have been outperformed by $\mathrm{CNN}$ based methods. Approaches to semantic segmentation exist for 2D raster images (Chen et al., 2018, Garcia-Garcia et al., 2017, He et al., 2017), 3D point clouds (Qi et al., 2017, Liu et al., 2017), and 3D volumetric representations (Riegler et al., 2017, Wang et al., 2017) as input, the latter ones however usually for scene part segmentation. While methods for volumetric data representations suffer from large memory consumption, $\mathrm{CNNs}$ for $3 \mathrm{D}$ point clouds need to tackle the challenge posed by the inherent lack of structure within this data representation. Methods aimed at 2D raster images are among the earliest works, yet the fusion step from two dimensional image data to 3D geometry has to be solved.

\section{CITY MODEL CREATION}

Despite its long research history, automatic 3D reconstruction may not produce results directly applicable to specific tasks. Simulating the shock wave propagation of an explosion within a complex 3D geometry is computationally demanding, where small, sparse, or mobile structures do not affect the result much or may not contribute relevant information while vastly increasing the computational complexity. Removing these structures manually is a time-consuming and hence infeasible task. We therefore augment the reconstruction process to automatically detect and remove unnecessary elements from the scene. To achieve this in a robust fashion, we propose a twofold approach: in a first step, we create a 3D reconstruction from georeferenced aerial images using conventional SfM and dense 3D matching methods. We then create semantic labels for all aerial images and fuse them with the dense point cloud obtained as an intermediate result of the previous step to create a semantic point cloud. The semantic information in is subsequently used to remove unnecessary scene information. The cleaned and preprocessed point cloud is then piped back into the SfM pipeline to create a meshed model of the scene.

\subsection{D Reconstruction}

In the most general sense, this task is solved by finding a large set of correspondences between images showing the same part of the scene, which can be used to solve a large system of equations with 3D locations of corresponding image points and camera pose parameters as variables. For the task of creating $3 \mathrm{D}$ reconstructions and preparing them for damage estimation of explosions, besides sufficient reconstruction quality, the output of the reconstructed point cloud together with point normal directions (information of the surface orientation related to the point location) and the refined camera poses (orientation and location in 3D) for each image are required. Since at this time several readily available software solutions solving this problem exist which do meet our requirements, we do not discuss the task of $3 \mathrm{D}$ reconstruction in greater detail.

\subsection{Semantic Fusion}

The geometry reconstruction step reconstructs all parts of the scene which were static during the time of image acquisition. These can be cars parked during that time, which do not add general information to an explosive expansion, as well as sparse 
or previous structures like trees and lampposts, which in practice do not alter the expansion of a shock wave significantly.To keep the task of numerical simulation of an explosive expansion within a complex geometry tractable, the geometry should be simplified by removing these structures. For an automated solution to this challenge, we propose using semantic information for each reconstructed 3D point to first separate the model into several classes relevant to the cleaning process and then process the parts of the model separately. The task of semantic segmentation can be paraphrased as the inference of some sort of sense for each data point directly from the data. It is usually defined in terms of a regression problem assigning each data point (each pixel in a 2D raster image or each point within a 3D point cloud) a label from a predefined set of class labels. The set of labels depends on the task to be solved, in this context we defined the semantic class label set $C=\{$ ground-level, wall, roof, vegetation, vehicles, clutter $\}$.

Since our 3D geometry model is generated from aerial images, we propose to perform semantic segmentation directly on these images and to fuse the semantic attributes later on with the 3D model. Besides the generally more robust performance of semantic segmentation methods on 2D images, we expect an advantage of added robustness due to the visibility of parts of the scene from different viewpoints. Since for our purpose a semantic resolution of the scene of several centimeters is sufficient, we propose to leverage an intermediate scene representation within an octree data structure for efficient and robust fusion of the semantic labels from all viewpoints with the 3D geometry. The core principal of the octree structure is the recurrent subdivision of a cubic volume into sub volumes of equal size, called nodes, up until a predefined target size of cubes at the lowest level, the so called leaf nodes, is reached. When inserting a point cloud model into the octree data structure, memory will only be allocated for leaf nodes containing points, which makes this representation extremely memory efficient and therefore suitable for the representation of large structures.

Methods for semantic segmentation produces for each data point a vector $l_{p}$ of real numbers of the same size as there are semantic classes, which are interpreted in terms of likelihoods of the data point belonging to the respective class. A semantic decision is usually made by assigning the label $c_{p}$ with the highest likelihood to the data point according to equation 1 , where $l_{p}(c)$ denotes the likelihood that pixel $p$ will belong to semantic class c.

$$
c_{p}=\underset{c}{\operatorname{argmax}} l_{p}(c)
$$

We postulate that in general, erroneous classifications will not be made by a large margin, therefore not casting direct class votes per view for a data point, but instead accumulating the likelihood vectors and taking the class with the maximum likelihood of the aggregate likelihood vector, should again produce more robust results.

We build our approach onto the open source octree implementation OctoMap (Hornung et al., 2013), which we augment to not only hold occupancy information for each node, but to also hold a likelihood vector $l_{n}$ per node encoding the likelihood of a node to belong to one of our semantic classes. Now, for each image, we cast a semantic vote per pixel by projecting a ray from the cameras center of projection into the octree starting from the camera location using the camera pose obtained during $3 \mathrm{D}$ reconstruction. We update the likelihood vector $l_{n}$ of the first occupied leaf node the ray intersects. We denote the set of pixels $p$ that contribute to a certain leaf node $n$ as $P_{n}$. After all source images have been processed, we assign a unique class label $c_{n}$ to each leaf node according to equation 2, and finally assign a label $c_{p} \in C$ to each point of the reconstructed model by querying the respective leaf node. We are now able to separate the model into parts according to their semantic class.

$$
c_{n}=\underset{c}{\operatorname{argmax}} l_{n}(c)=\underset{c}{\operatorname{argmax}} \sum_{p \in P_{n}} l_{p}(c)
$$

\subsection{Model Cleaning}

Suitable approaches for cleaning irrelevant parts from the scene depend on their class. Whereas usually small structures from the class clutter, such as posts, benches, signal masts, and lampposts can be removed directly without negatively impairing the scene integrity, for larger structures (cars from the class vehicle, trees from the class vegetation) a different approach is to be pursuit. Cars, especially on parking areas, may form larger patches within the reconstruction, directly removing them may result in holes in the model or higher complexity in meshing. Trees on the other hand may cause problems in case of limited training data, which can lead to meadows, which are part of the groundlevel category which we want to keep, being erroneously classified as trees. In both cases, we leverage an estimate of the local height of the ground level, which is extracted by rasterizing the point cloud in the xy-plane (assuming a coordinate system in which the $\mathrm{z}$-axis is antiparralel to the direction of gravity, i.e., it points in the "up" direction) and using the top-hat filter to estimate the ground level ( $\mathrm{Li}$ et al., 2014). An alternative approach for ground level estimation working directly on point clouds may be cloth-simulation (Zhang et al., 2016).

Now, prior to removal of points segmented as vehicles, they are partitioned into single clusters based on the euclidean distance between points. Then the points for each vehicle cluster are projected onto the ground level estimate for that area together with the ground-level points surrounding the respective cluster within a distance $d$. This "padding" is done to account for sloping effects arising from the reconstruction around vehicles. For points in the semantic class vegetation, in a first step the labels of points close to the estimated ground level are corrected to the class ground-level and the remaining points in vegetation are clustered based on their euclidean distance. Small clusters can be removed directly, larger clusters are processed in the same way as the clusters for vehicles. For all altered points, the point normal vectors are update with the corresponding ones extracted from the ground level estimate.

Finally, the cleaned point cloud is then returned into the 3D reconstruction pipeline to create a meshed wire frame model which can be used as geometry for numerical simulation.

\section{EXPLOSION SIMULATION}

The estimation of the resulting blast wave propagation of a predefined charge size within the geometry model is solved with CFD numerical simulation.

\subsection{Numerical setup}

We use a transient Reynolds-Averaged Navier-Stokes (RANS) simulation for our numerical investigations applying the commercial finite volume solver ANSYS $\AA$ Fluent $\AA$. Because of 
high shock velocities (Mach number $M a>0.3$ ) occurring in an explosion, their impact on the density can not be neglected, so the fluid density varies according to the ideal gas law. As a fluid, air is defined as an ideal gas, it is assumed that air behaves like a standard compressible fluid with an inviscuous flow. Using this inviscid flow assumption, the impact of fluid viscosity in the model can be neglected. When viscous forces are neglected, the Navier-Stokes equation can be simplified to a form known as the Euler equation. Furthermore, according to the boundary conditions (Figure 2), the houses and floor areas of the $3 \mathrm{D}$ geometry have been defined as free-slip walls and the environment as a non-reflecting total pressure with total ambient pressure of $1 \mathrm{~atm}$. Due to the complexity in the 3D model, we do not resolve deformations or the destruction of the geometry, it is therefore assumed to be rigid. Once a virtual ex-

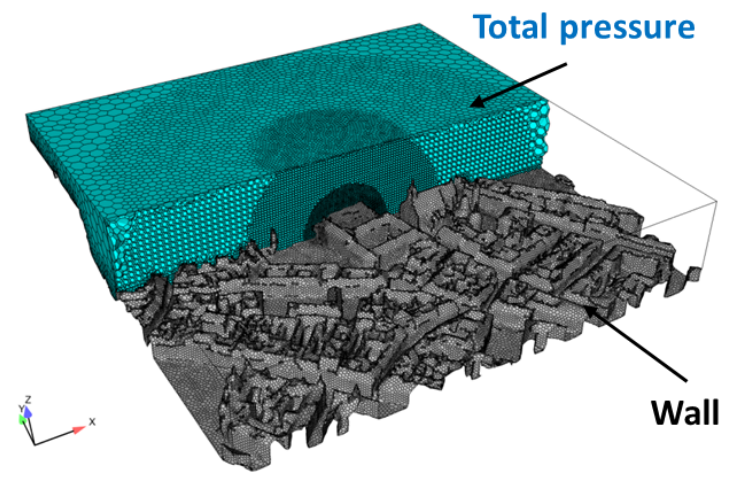

Figure 2. Polyhedral mesh based on the imported 3D model. The volumetric resolution is at its finest level around the blast center and gets coarser with increasing distance.

plosive charge has been placed in the geometry (cf. Figure 6 in Section 6), we discretized the whole fluid domain applying a volumetric polyhedral meshing strategy. To achieve an accurate solution from the simulation, a fine volumetric resolution of the mesh is needed in areas of high local pressure gradients (Figure 2). The highest resolution mesh zone starts in the near region of the explosive charge where the highest pressure gradients are occurring. The consequence of the explosion is a high intensity wave that spreads from the source outwards to the surrounding air. As the wave propagates, it decreases in pressure and speed with increasing distance from the center of the explosion (Figure 3). Thus, the volumetric resolution of the mesh can be decreased with increasing distance to the detonation origin in accordance with the dropping pressure gradients. This meshing strategy provides high resolution of the blast wave simulation at any time and location while keeping the computational cost low.

\subsection{Model of an explosive charge as compressed gas}

We model the explosive charge as a spherical volume of compressed gas with high over pressure, which acts as the source of the explosion. In the model, the radius of the sphere is set to 1 meter, the internal energy of the containing compressed gas is the same as the energy released by the explosive charge. To simulate the blast wave propagation for a given charge size, we set the expected pressure for this initial volume using the equation (3) according to Brode (Fisher, Schelyaev, 2017).

$$
p_{\text {Brode }}=\frac{E_{T N T}(\gamma-1)}{V_{b a l}}+p_{0},
$$

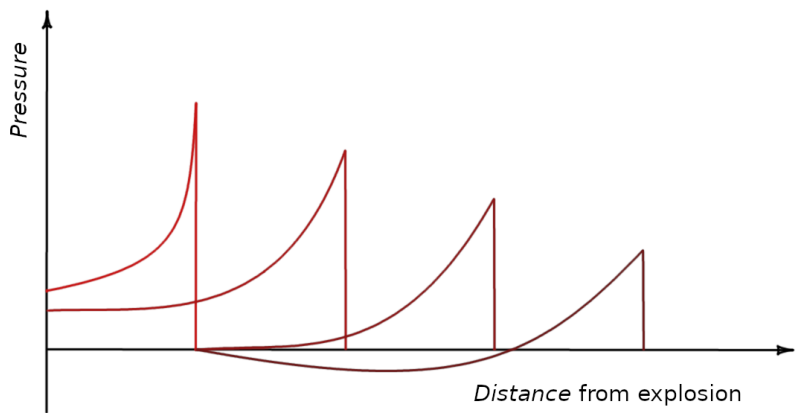

Figure 3. Blast pressure as a function of distance at four points in time, reproduced according to (Ngo et al., 2007).

For the calculation, compressible air is used as a fluid. The compression is adiabatic (ideal gas), therefore the compressed gas inside the volume has the same properties as air at a specific temperature and pressure. We define the energy released by an explosive charge in terms of TNT equivalents. This energy is necessary to calculate the magnitude of the momentum of the explosion, and calculated from the equivalent TNT mass $M_{T N T}$ according to equation (4) (Fisher, Schelyaev, 2017).

$$
E_{T N T}=M_{T N T} \cdot 4,52 \cdot 10^{6}
$$

The adiabatic mathematical expression (5) determines the resulting temperature of the compressed gas with initial pressure $p_{\text {Brode }}$ inside the sphere (Fisher, Schelyaev, 2017).

$$
T=T_{0}\left(\frac{p_{\text {Brode }}}{p_{0}}\right)^{\frac{\gamma-1}{\gamma}}
$$

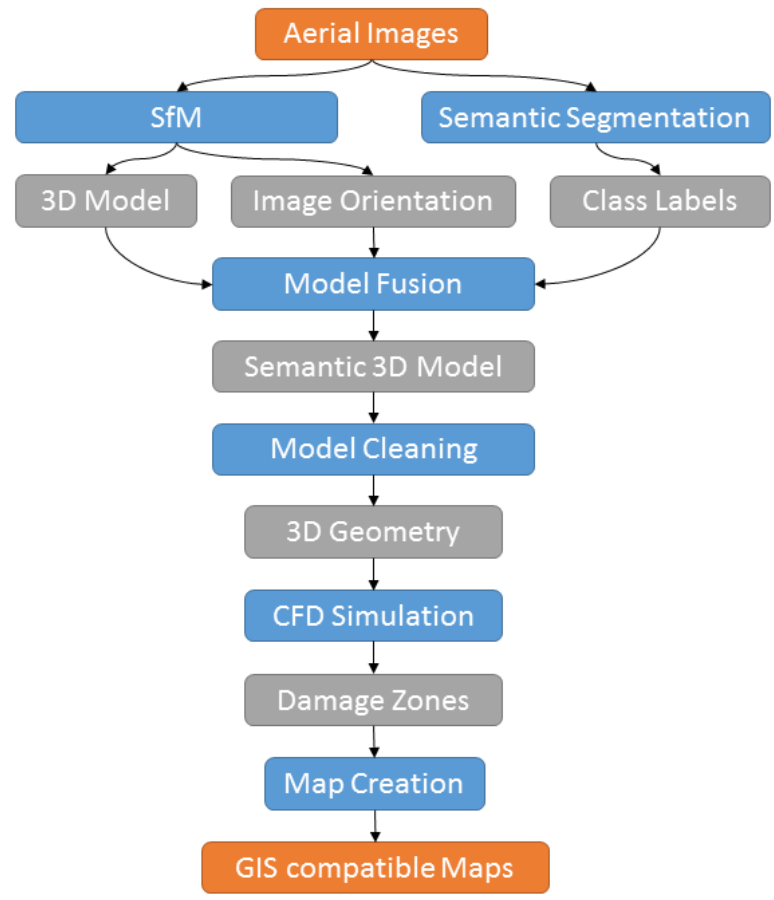

Figure 4. Schematic overview of the workflow. Orange boxes depict input and output data, grey boxes mark intermediate results and blue boxes symbolize specific modules in our pipeline. 


\section{INTERPRETATION AND UTILIZATION}

The local maximum in calculated blast pressure enables estimations of the effects occurring in different regions of the domain. With this information studies on environmental impacts and consequences for the human population can be carried out.

Table 1. Blast over pressures and associated maximum wind speed effect on various structures and the human body according to (Glasstone, Dolan, 1977, Sartori, 1983).

\begin{tabular}{|c|c|c|c|}
\hline $\begin{array}{l}\text { peak over } \\
\text { pressure }\end{array}$ & $\begin{array}{l}\text { maximum } \\
\text { wind speed }\end{array}$ & effect & effect on the human body \\
\hline $1 \mathrm{psi}$ & $60 \mathrm{kph}$ & window glass shatters & $\begin{array}{l}\text { light injuries from frag- } \\
\text { ments occur }\end{array}$ \\
\hline $2 \mathrm{psi}$ & $110 \mathrm{kph}$ & $\begin{array}{l}\text { moderate damage to } \\
\text { houses (windows and } \\
\text { doors blown out, severe } \\
\text { damage to roofs) }\end{array}$ & $\begin{array}{l}\text { people injured by flying } \\
\text { glass and debris }\end{array}$ \\
\hline 3 psi & $165 \mathrm{kph}$ & $\begin{array}{l}\text { residential structures col- } \\
\text { lapse }\end{array}$ & $\begin{array}{l}\text { serious injuries are com- } \\
\text { mon, fatalities may occur }\end{array}$ \\
\hline $5 \mathrm{psi}$ & $265 \mathrm{kph}$ & most buildings collapse & $\begin{array}{l}\text { injuries are universal, } \\
\text { fatalities are widespread }\end{array}$ \\
\hline $10 \mathrm{psi}$ & $475 \mathrm{kph}$ & $\begin{array}{l}\text { reinforced concrete } \\
\text { buildings are severely } \\
\text { damaged or demolished }\end{array}$ & most people are killed \\
\hline $20 \mathrm{psi}$ & $800 \mathrm{kph}$ & $\begin{array}{l}\text { heavily built concrete } \\
\text { buildings are severely } \\
\text { damaged or demolished }\end{array}$ & fatalities approach $100 \%$ \\
\hline
\end{tabular}

Table 1 provides an estimate of the effects of increasing blast pressure on structures and people based on data of the Department of Defense study (Glasstone, Dolan, 1977) conducted by Glasstone and Dolan. Due to the estimation provided by the explosion simulation that the local maximum blast over pressure will reach or surpasses specific limits in a given scenario, damaged or demolished concrete buildings and injuries or even fatalities in the population can be expected in certain areas.

Using this damage classification in combination with the results from the explosion simulation, we propose to create map layers for GIS applications detailing damage zones in the following ways:

- Map layers showing the detailed damage zones according to the damage classes of Table 1. These will provide insight in possibly unexpected disconnected areas further away from the blast center with again heightened pressure. These occur due to interference effects of multiple wave fronts stemming from reflections at building surfaces. We postulate that this information can heighten the understanding of wave propagation effects and channeling effects in urban environments occurring during explosions.

- Map layers showing the maximum areas in which certain damages can be expected according to the simulation in terms of a convex hull around all areas of that specific damage class. This provides insight into the difference in spread between over pressure ranges as well as between different charge sizes. With this information it is also possible to determine secure locations to position security and rescue forces in the run-up of an event which is expected to face a heightened threat level.

- For geoinformation systems capable of showing changes over time we can prepare this information not in terms of the overall expected maximum pressure but as a time series showing the expansion and ddevelopment of pressure areas, again aiding in the qualitative understanding of the effects of blast pressure propagation in training events for disaster response personnel.

- This information can also be provided with full 3D information to be integrated into personnel training, again adding to the qualitative understanding of effects to be expected in blast wave propagations in urban environments.

Furthermore, if it is possible to enrich the geometry model with additional information, possibly drawn from existing land registers, even more detailed damage analysis can be done. We will show this exemplary during our case study detailed in section 6 by first detecting windows in terrestrial imagery registered to our geometry model and analysing different scenarios in terms of expected glass damage.

\section{EXPERIMENTS AND CASE STUDY}

To demonstrate our proposed approach, we processed a reconstruction of a square portion with an edge length of ca. 500 meters of the inner city of Graz, Austria, around a square chosen as our target area for placement of our virtual explosives. The resulting point cloud model consists of 75 million points with an average distance of 10 centimeters. Since no suitable data set for semantic segmentation of aerial images into classes relevant to our task was available, we partially annotated 4 out of a total of 345 source images by hand. For semantic segmentation we used the DeepLabV3+ network architecture of (Chen et al., 2018), retraining their Xception65 architecture model pretrained on the CityScapes autonomous drivingdata sett (Cordts et al., 2016) on our manually annotated data.

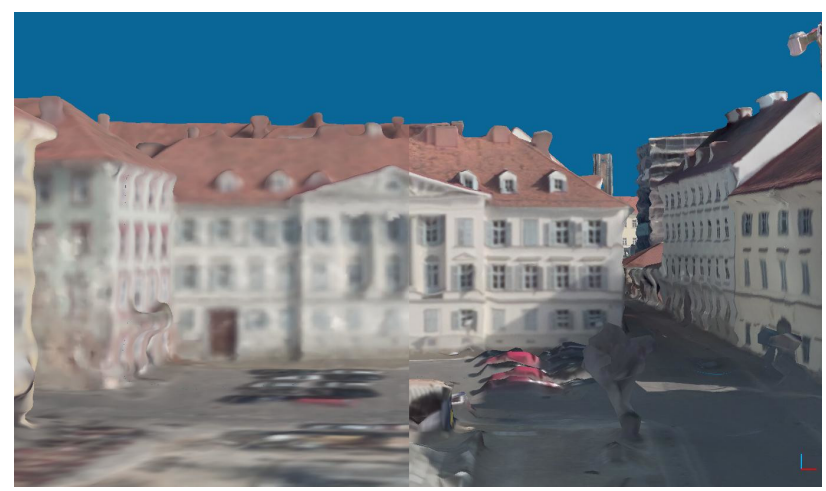

Figure 5. Comparison of the cleaned (but untextured) 3D geometry on the left with the raw, textured 3D reconstruction on the right. Cars, trees, and elements of street infrastructure (class

clutter), visible on the right, are missing on the left side.

Fusion of the 3D reconstruction with the 2D semantic segmentations on the aerial images was done using the approach described in section 3.2 with our customized OctoMap using a leaf node size of $20 \mathrm{~cm}$. After automatic segmentation, a few areas $(<10)$ had to be corrected manually due erroneous semantic labeling consistent in all aerial views. We were able to do this by applying simple handcrafted filters based on heuristics such as height over ground level and orientation of the normal vector relative to the $\mathrm{z}$-axis in those areas.

As a last step of processing the point cloud before meshing and simulation, we cleaned it using the algorithm outlined in 3.3. 
Figure 5 shows a comparative view of the 3D model before and after cleaning.

We used the meshing tool ANSYS $₫$ ICEM $\cap$ to prepare the geometry to obtain the appropriate surface model for generating the numerical mesh within a single fluid domain. The explosives were placed near a square in the center of the reconstructed 3D geometry (cf. Figure 6), the simulation was performed four times with charge sizes of $1 \mathrm{~kg}, 10 \mathrm{~kg}, 100 \mathrm{~kg}$ and $1000 \mathrm{~kg}$ of TNT equivalents, respectively.

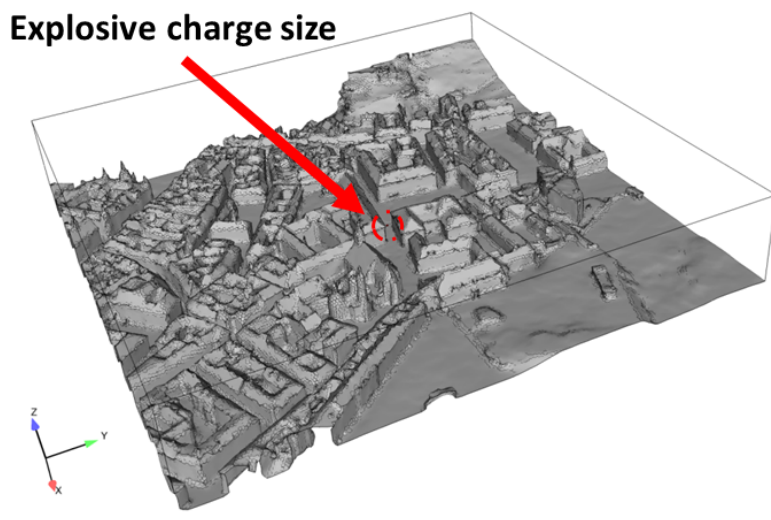

Figure 6. Location of the explosive charge in the center of the city.

The numerical results provide a complete pressure estimation of the time course for the expected blast wave propagation. Exemplary, the expansion of the blast wave is shown at four points in time for the scenario of $1000 \mathrm{~kg}$ of TNT equivalent charge in Figure 7.

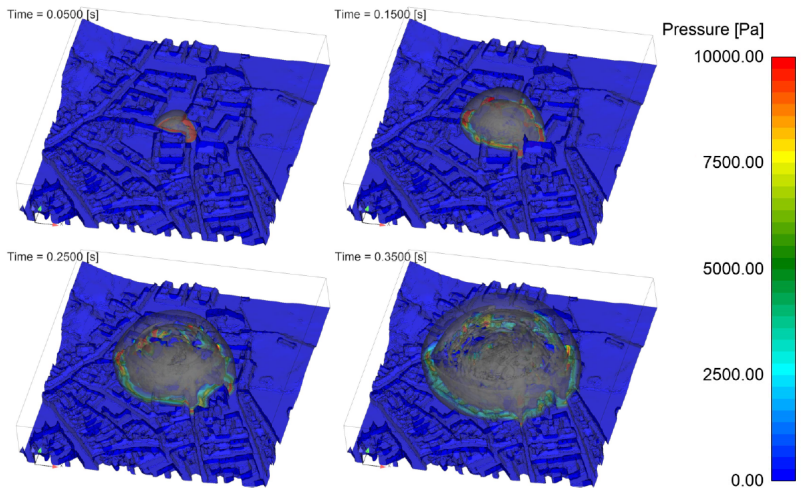

Figure 7. Estimation of the expected pressure propagation over time for $1000 \mathrm{~kg}$ of TNT equivalent.

Using this result, we can query the areas at which a certain pressure is reached or surpassed, generating map layers showing the different damage zones for the specific scenario on top of other GIS sources. Figure 8 shows a comparison of the damage zones for scenarios of $10 \mathrm{~kg}$ and $100 \mathrm{~kg}$ TNT equivalent charge, overlayed on the street map (map data courtesy of OpenStreetMap) in QGIS. It is apparent from the comparison of the maps that the damage radius does not scale linearly, neither for the area where a certain absolute maximum pressure is reached or surpassed, nor for the maximum propagation. Channeling effects due to urban canyons are visible. In the detailed view in Figure 9, unexpected areas of higher pressure comparatively far from the center of the explosion, beyond ares with lower maximum pressure, are visible. These effects occur due to deflection and ram

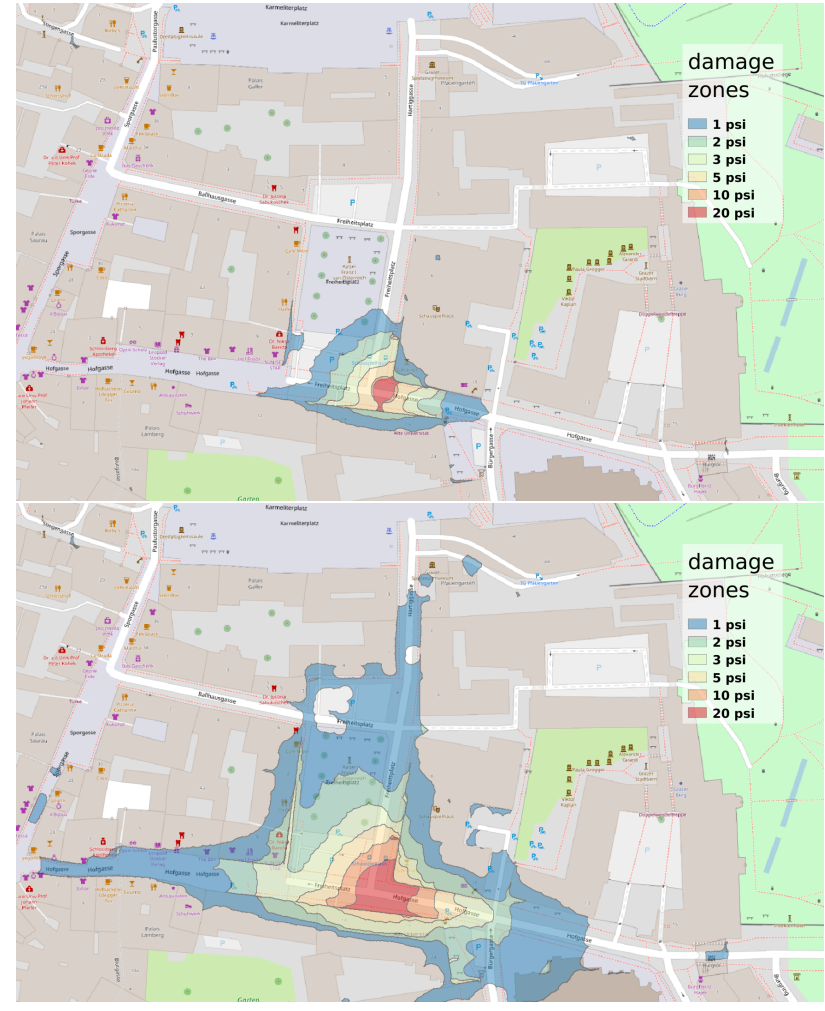

Figure 8. Comparison of damage zones overlayed on a street map for charge sizes of $10 \mathrm{~kg}$ (top) and $100 \mathrm{~kg}$ (bottom).

pressure effects caused by buildings.

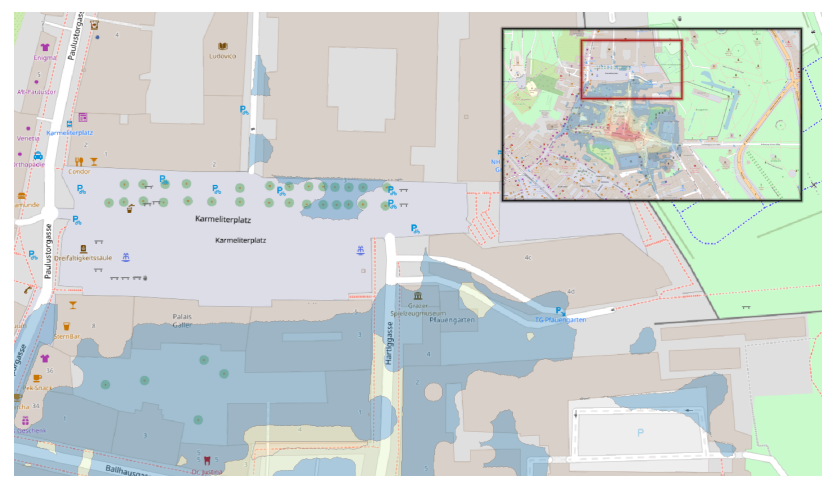

Figure 9. Detailed view of the damage zone for the scenario of a $1000 \mathrm{~kg}$ explosive charge. The simulation reveals disconnected areas of expected glass damage (blue areas) far away from the center of the detonation.

A more robust evaluation can be seen in figure 10 for the case of $10 \mathrm{~kg}$ of TNT equivalent, where the damage zones are shown as the convex hull over all areas of a certain damage zone, again overlayed over the street map in QGIS.

As a quantitative analysis, Table 2 compares the total area of the destruction zones of all four scenarios. Again, a non-linear relation between charge size and affected area, as well as between maxima in reached pressure, are visible.

As a further application of the explosion simulation, we evaluate the amount of expected window breakage in the direct vicinity of the square where the virtual charges have been placed. Towards that task, we started by detecting windows on the building facades in terrestrial images registered to our 3D reconstruc- 


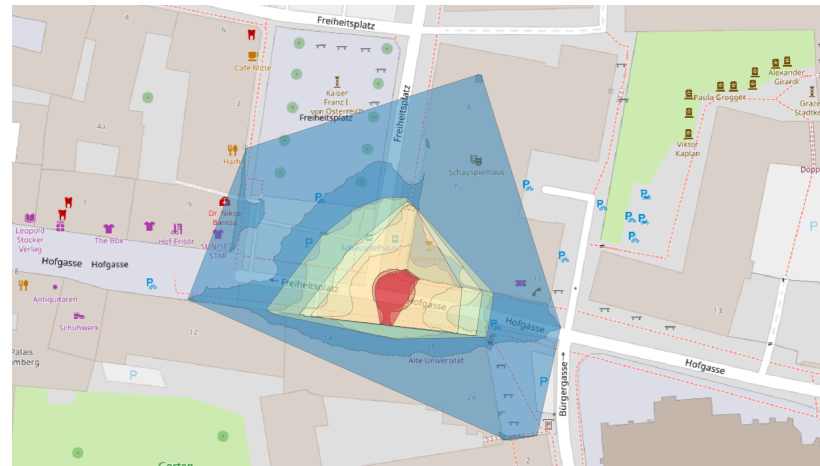

Figure 10. Comparison of the depiction of the convex hull of the damage zones with their original estimate for the case of $10 \mathrm{~kg}$ equivalent charge size.

Table 2. Areas in $m^{2}$ within destruction zones depending on the charge size

\begin{tabular}{rrrrr} 
peak pressure & $1 \mathrm{~kg}$ & $10 \mathrm{~kg}$ & $100 \mathrm{~kg}$ & $1000 \mathrm{~kg}$ \\
\hline $1 \mathrm{psi}$ & 505 & 2370 & 12700 & 53500 \\
$2 \mathrm{psi}$ & 270 & 1160 & 5120 & 23000 \\
$3 \mathrm{psi}$ & 185 & 690 & 2880 & 15800 \\
$5 \mathrm{psi}$ & 70 & 370 & 1490 & 7380 \\
$10 \mathrm{psi}$ & 15 & 100 & 830 & 3750 \\
$20 \mathrm{psi}$ & 3 & 90 & 420 & 1650
\end{tabular}

tion. Again, we retrained a DeepLabV3+ segmentation network pre-trained on the CityScapes autonomous driving corpus on a few manually annotated training images taken of the facades surrounding the square. Fusing the window detections with the reconstructed city model as detailed in section 3.2 (using a semantic resolution of $15 \mathrm{~cm}$ ) allows us to determine their georeferenced locations and thereby to correlate their positions with the respective damage zones corresponding to glass damage in the four simulated scenarios. In total, 367 windows were de-

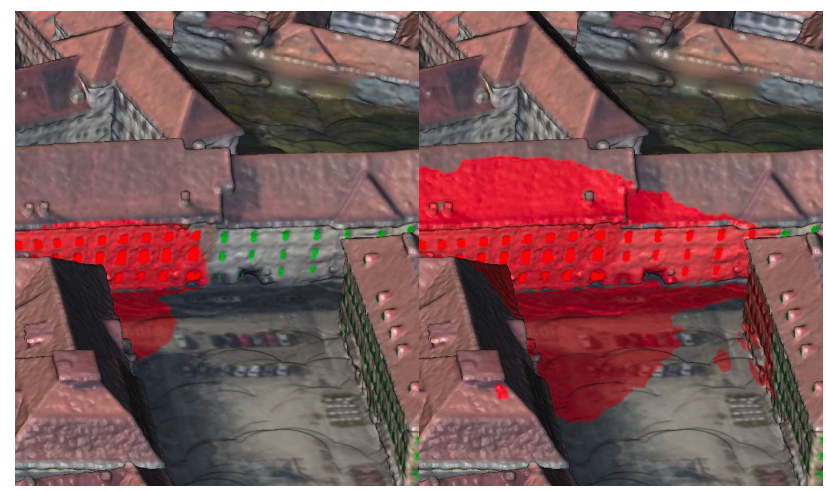

Figure 11. A comparison of the damage zones of 1 psi on a facade. The damage zone is overlayed in red on the geometry, broken windows are highlighted in bright red, those expected to survive the blast in green. On the left side the scenario of $1 \mathrm{~kg}$

TNT, on the right side that of $10 \mathrm{~kg}$ TNT is shown.

tected around the square. In accordance with the damage classification of (Glasstone, Dolan, 1977) (cf. Table 1), windows less than 0.5 meters away from the 1 psi pressure zone were assumed to be broken. Table 3 compares the expected number of broken windows around the square and the total area of glass broken by the explosion of the respective charge size. Con-
Table 3. Number and glass area of broken windows in each scenario of a total of 367 windows detected around the square.

\begin{tabular}{lrrrr} 
& $1 \mathrm{~kg}$ & $10 \mathrm{~kg}$ & $100 \mathrm{~kg}$ & $1000 \mathrm{~kg}$ \\
\hline broken windows & 48 & 105 & 319 & 363 \\
broken glass $\left[\mathrm{m}^{2}\right]$ & 115 & 235 & 630 & 685
\end{tabular}

cerning the comparatively small increase from 319 to 363 probably destroyed windows it has to be noted that only windows around the square were detected, yet the $1 \mathrm{psi}$ pressure range of the blast wave resulting from the $100 \mathrm{~kg}$ scenario fills the square already almost completely, while the one from the 1000 $\mathrm{kg}$ scenario outreaches it by far. Figure 11 shows the qualitative difference of the number of affected windows depending on charge sizes on selected facades for the scenario of $1 \mathrm{~kg}$ of TNT equivalent charge size on the left and the scenario of $10 \mathrm{~kg}$ of TNT equivalent charge size on the right. Windows outside the 1 psi pressure range are highlighted in green, the ones within the damage zone are marked in bright red.

\section{CONCLUSION}

In this work, we proposed a methodology for creating reconstructions of urban areas from aerial and terrestrial images with automated preprocessing for use as geometry in numerical simulation of the impact of explosions in urban environments. We showed how the results can be used to create GIS compatible maps of areas where certain damages can be expected in different threat scenarios which can be directly integrated into existing geographic information systems. These maps may then be used in training of personnel involved in disaster management or for preparation and planning in the fore run of major events.

Due to the time effort needed (adequate source images or a geometry model must be available, the numerical simulation of a single explosive expansion takes depending on the charge size up to several days) the application is still constrained to the creation of training scenarios or in planning of major events. An immediate application in an unforeseen crisis is not possible.

Furthermore, real-world experiments verifying the damage predictions may prove insightful, since the damage classification used stems from an american study more than 40 years old, where building standards and therefore damage patterns may not be directly applicable to central Europe.

\section{ACKNOWLEDGEMENTS}

Work on this paper has been funded by the Austrian Research Promotion Agency (FFG) with the projects no. 860661 and no. 855331.

Geo-referenced aerial and terrestrial images courtesy of Meixner Vermessung ZT GmbH, Linke Wienzeile 4, 1060 Wien, Austria.

\section{REFERENCES}

Agisoft, 2019. Agisoft Metashape User Manual. Agisoft LLC.

Brode, H. L., 1955. Numerical solutions of spherical blast waves. Journal of Applied Physics, 26(6), 766-755.

Chen, L.-C., Zhu, Y., Papandreou, G., Schroff, F., Adam, H., 2018. Encoder-decoder with atrous separable convolution for semantic image segmentation. ECCV. 
Cordts, M., Omran, M., Ramos, S., Rehfeld, T., Enzweiler, M., Benenson, R., Franke, U., Roth, S., Schiele, B., 2016. The cityscapes dataset for semantic urban scene understanding. Proc. of the IEEE Conference on Computer Vision and Pattern Recognition (CVPR).

Fairlie, G., 1998. The numerical simulation of high explosives using autodyn-2d \& 3d. Institute of Explosive Engineers 4th Biannual Symposium, 743-751.

Fisher, Y., Schelyaev, A. E., 2017. Simulation of explosions in flowvision cfd software. Technical report, FlowVision.

Garcia-Garcia, A., Orts-Escolano, S., Oprea, S., VillenaMartinez, V., Garcia-Rodriguez, J., 2017. A review on deep learning techniques applied to semantic segmentation. arXiv preprint arXiv:1704.06857.

Glasstone, S., Dolan, P. J., 1977. The effects of nuclear weapons. Technical report, U.S. Department of Defense and the Energy Research and Development Administration.

Hartley, R., Zisserman, A., 2000. Multiple View Geometry in Computer Vision. Cambridge University Press, Cambridge, U.K.

He, K., Gkioxari, G., Dollár, P., Girshick, R., 2017. Mask r-cnn. Proceedings of the IEEE international conference on computer vision, 2961-2969.

Hornung, A., Wurm, K., Bennewitz, M., Stachniss, C., Burgard, W., 2013. OctoMap: An Efficient Probabilistic 3D Mapping Framework Based on Octrees. Autonomous Robots. http://octomap.github.com. Software available at http://octomap.github.com.

Larcher, M., Casadei, F., 2010. Explosions in complex geometries - a comparison of several approaches.

Li, Y., Yong, B., Wu, H., An, R., Xu, H., 2014. An improved top-hat filter with sloped brim for extracting ground points from airborne lidar point clouds. Remote sensing, 6(12), 1288512908.

Liu, F., Li, S., Zhang, L., Zhou, C., Ye, R., Wang, Y., Lu, J., 2017. 3dcnn-dqn-rnn: A deep reinforcement learning framework for semantic parsing of large-scale $3 \mathrm{~d}$ point clouds. The IEEE International Conference on Computer Vision (ICCV).

Mapillary, 2017. OpenSfM. https://www.opensfm.org/.

Ngo, T., Mendis, P., Gupta, A., Ramsay, J., 2007. Blast Loading and Blast Effects on Structures. EJSE Special Issue: Loading on Structures.

Özyeşil, O., Voroninski, V., Basri, R., Singer, A., 2017. A survey of structure from motion*. Acta Numerica, 26, 305-364.

Qi, C. R., Yi, L., Su, H., Guibas, L. J., 2017. Pointnet++: Deep hierarchical feature learning on point sets in a metric space. Advances in neural information processing systems, 5099-5108.

Riegler, G., Osman Ulusoy, A., Geiger, A., 2017. Octnet: Learning deep $3 \mathrm{~d}$ representations at high resolutions. Proceedings of the IEEE Conference on Computer Vision and Pattern Recognition, 3577-3586.

Sartori, L., 1983. The effects of nuclear weapons. Physics Today, 32-41.
Schönberger, J. L., Frahm, J.-M., 2016. Structure-from-Motion Revisited. Conference on Computer Vision and Pattern Recognition (CVPR).

Sohaimi, A. S., Risby, M., Ishak, S. A., Khalis, S., Norazman, M., Ariffin, I., Yusof, M., 2016. Using Computational Fluid Dynamics (CFD) for Blast Wave Propagation under Structure. Procedia Computer Science, 80, 1202-1211.

Wang, P.-S., Liu, Y., Guo, Y.-X., Sun, C.-Y., Tong, X., 2017. Ocnn: Octree-based convolutional neural networks for $3 \mathrm{~d}$ shape analysis. ACM Transactions on Graphics (TOG), 36(4), 72.

Zhang, W., Qi, J., Wan, P., Wang, H., Xie, D., Wang, X., Yan, G., 2016. An easy-to-use airborne LiDAR data filtering method based on cloth simulation. Remote Sensing, 8(6), 501. 